01

\title{
Низковольтный мембранный интерфейс для экстракции ионов из полярных растворов
}

\author{
() А.А. Балакин, Е.А. Буйдо \\ Филиал Института энергетических проблем химической фризики им. В.Л. Тальрозе РАН, \\ 142432 Черноголовка, Московская обл., Россия \\ e-mail: balakin@binep.ac.ru
}

(Поступило в Редакцию 18 декабря 2017 г.)

\begin{abstract}
Проведены численные расчеты напряженности электрического поля, экстрагирующего ионы из полярных растворов в паровую фазу в мембранном интерфейсе с электродом, расположенным непосредственно на вакуумной поверхности мембраны. Такая конструкция позволит существенно снизить напряжение, способное обеспечить эффективную эмиссию ионов из раствора, заполняющего канал мембраны, что даст возможность улучшить характеристики мембранного интерфейса.
\end{abstract}

DOI: 10.21883/JTF.2018.08.46303.2603

\section{Введение}

Интерес к изучению полевой экстракции ионов из жидкости связан с тем, что этот процесс является ключевым в методиках генерации газофазных ионов слаболетучих веществ, широко используемых в массспектральном анализе растворов. Такой способ непосредственного перевода ионов из раствора в газовую фазу не сопровождается разрушением исходной структуры имеющихся в растворе ионов, что позволяет использовать его как метод мягкой ионизации молекул различной природы. Для эффективного выхода ионов из полярной жидкости требуются электрические поля напряженностью порядка $10 \mathrm{MV} / \mathrm{cm}$ и более [1,2].

Впервые возможность создания условий для прямого полевого испарения ионов из полярного раствора без его разбрызгивания была продемонстрирована в [3]. Стабилизация поверхности жидкости в сильном электрическом поле обеспечивалась за счет того, что содержащий ионы раствор помещали в каналы полимерной трековой мембраны толщиной около $10 \mu \mathrm{m}$, диаметр которых составлял несколько десятков нанометров. В стационарных условиях сильное локальное электрическое поле, способное стимулировать достаточно эффективный выход ионов из полярной жидкости, создавалось благодаря зарядке поверхности диэлектрической мембраны $[1,4,5]$. Однако эта методика экстракции ионов из растворов имеет ряд существенных недостатков, ограничивающих ее использование, в частности, в аналитических приложениях. Это связано с тем, что в использовавшейся методике нет возможности прямого управления величиной поверхностного заряда на вакуумной стороне мембраны, а также существует его зависимость от тока ионов, выходящих из раствора.

Эти недостатки можно преодолеть, если использовать кратковременно действующее электрическое поле. В таком режиме из-за большой разницы в электропроводности раствора и полимерной основы мембраны сильное электрическое поле у поверхности раствора, заполняющего каналы мембраны, обеспечивается за счет эффекта острия [6]. При этом его напряженность будет пропорциональна напряжению между раствором и экстрагирующим электродом, что в отличие от стационарного режима делает выход ионов управляемым. Действительно, экспериментальная проверка возможности реализации такого режима $[7,8]$ показала, что стабильную экстракцию ионов из водно-глицериновой смеси можно получить, прикладывая импульсы высокого напряжения (более $5 \mathrm{kV}$ при расстоянии между мембраной и электродом в вакууме около $0.2 \mathrm{~mm}$ ). Вместе с тем было установлено, что в генерируемом источником ионном пучке наряду с ионами, выходящими из раствора, присутствуют ионы, возникающие в процессах вторичной эмиссии при бомбардировке мембраны и электрода в вакууме экстрагируемыми из раствора первичными ионами, ускоренными до энергии в несколько килоэлектронвольт.

Влияние вторичных процессов может быть существенно уменьшено, если экстрагировать ионы более низким напряжением. При этом необходимо сохранить напряженность электрического поля у поверхности раствора, заполняющего каналы мембраны, порядка $10 \mathrm{MV} / \mathrm{cm}$. Вместе с тем уменьшение зазора между мембраной и электродом в вакууме вряд ли возможно в конструкции, использовавшейся в $[7,8]$. Другой подход состоит в том, чтобы экстрагирующий электрод перенести на вакуумную поверхность мембраны. Такая конструкция модифицированного мембранного интерфейса была предложена в [9].

Цель настоящей работы состоит в том, чтобы определить параметры модифицированного мембранного интерфейса, с помощью которого можно обеспечить эффективную полевую экстракцию ионов из полярных растворов, используя экстрагирующее напряжение порядка $100 \mathrm{~V}$. Для этого проведены модельные расчеты напряженности электрического поля, экстрагирующего ионы из раствора, заполняющего каналы мембраны, при различных геометрических параметрах системы. 


\section{Модель и результаты расчетов}

Исследуемый раствор находится на внешней стороне лавсановой трековой мембраны, которая разделяет атмосферную и вакуумную части интерфейса. Для создания электрического поля, стимулирующего выход ионов из раствора, к электродам (погруженному в раствор и экстрагирующему, расположенному на вакуумной стороне мембраны) прикладывается напряжение. Между электродом на вакуумной стороне мембраны и каналом, заполненным раствором, имеется диэлектрический зазор. Конфигурация модифицированного мембранного интерфейса показана на рис. 1.

В расчетах использовалась аксиально-симметричная модель, показанная на рис. 2, ось которой совпадает с осью канала в мембране.

Напряженность электрического поля на оси канала вблизи поверхности раствора определялась с помощью программы SIMION 8 [10]. При этом для упрощения принималось, что раствор, заполняющий канал мембраны, является проводником. Кроме того, принималось, что диэлектрическая постоянная материала мембраны равна единице. В расчетах варьируемыми параметрами были (рис. 2): толщина электрода на вакуумной поверхности мембраны $(\Delta)$, ширина диэлектрического кольца

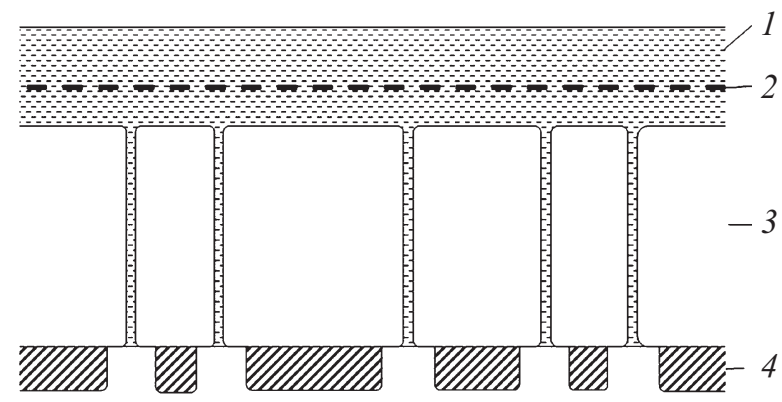

Рис. 1. Схема расположения элементов мембранного интерфейса: 1 - раствор, 2 - электрод в растворе, 3 - полимерная мембрана с каналами, 4 - электрод на вакуумной стороне мембраны.

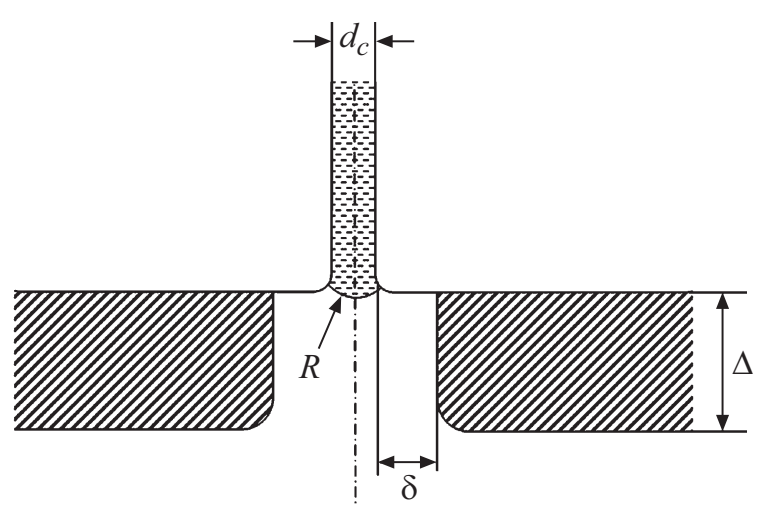

Рис. 2. Модель и параметры для расчетов напряженности электрического поля на оси канала вблизи поверхности раствора.

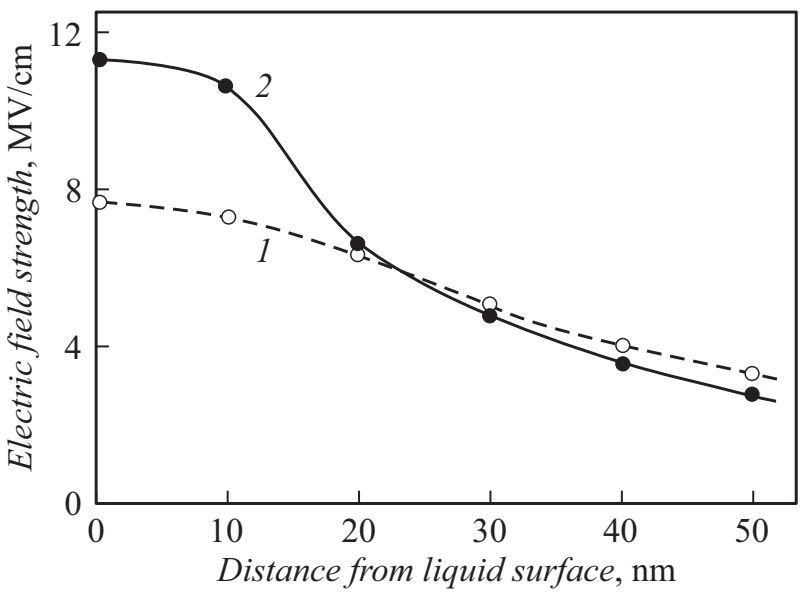

Рис. 3. Напряженность электрического поля в зависимости от расстояния до поверхности раствора в канале мембраны для плоской (1) и сферической (2) поверхностей раствора.

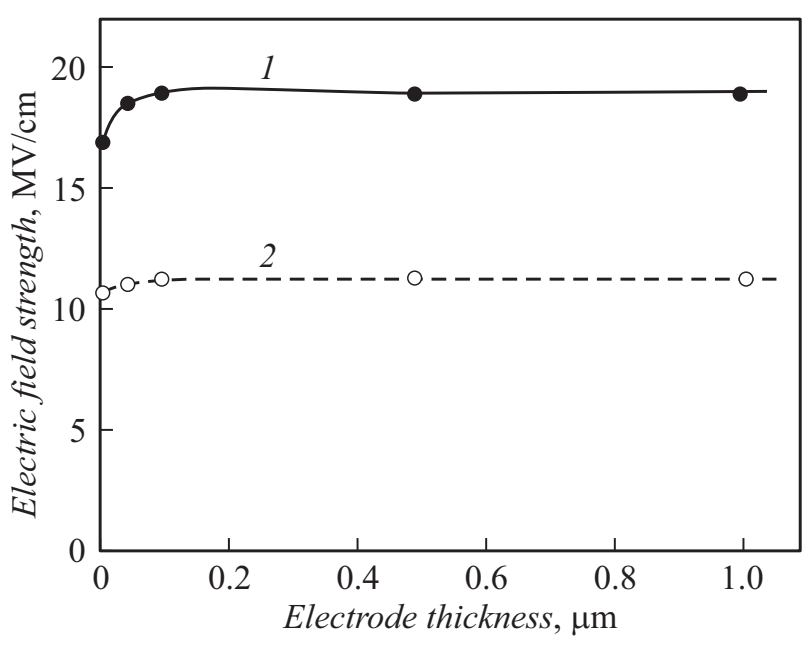

Рис. 4. Зависимость напряженности электрического поля у поверхности раствора от толщины электрода на поверхности мембраны при $\delta=0.02$ (1) и $\delta=0.22 \mu \mathrm{m}(2)$.

вокруг канала, которое разделяет раствор и электрод на поверхности мембраны $(\delta)$, а также радиус кривизны поверхности раствора на вакуумной стороне $(R)$. Во всех расчетах диаметр канала $d_{c}$ принимался равным $60 \mathrm{~nm}$. Область расчетов по радиусу ограничивалась $1.5 \mu \mathrm{m}$, что составляет половину среднего расстояния между каналами при плотности каналов на поверхности мембраны $10^{7} \mathrm{~cm}^{-2}$. Значения этих параметров близки к значениям для мембран, использовавшихся в реальных экспериментах [7,8]. Напряжение между раствором и электродом на вакуумной поверхности мембраны принималось равным $50 \mathrm{~V}$.

На рис. 3 представлены зависимости напряженности электрического поля от расстояния до поверхности раствора при толщине электрода $\Delta=0.5 \mu \mathrm{m}$ и ширине диэлектрического зазора $\delta=0.22 \mu \mathrm{m}$ для двух случаев: 


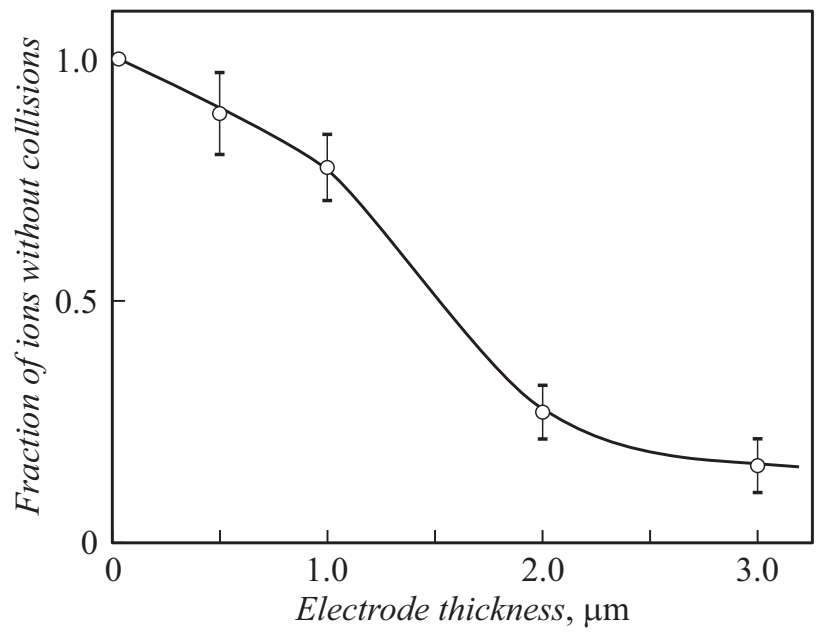

Рис. 5. Доля ионов, избежавших соударений с экстрагирующим электродом, в зависимости от его толщины.

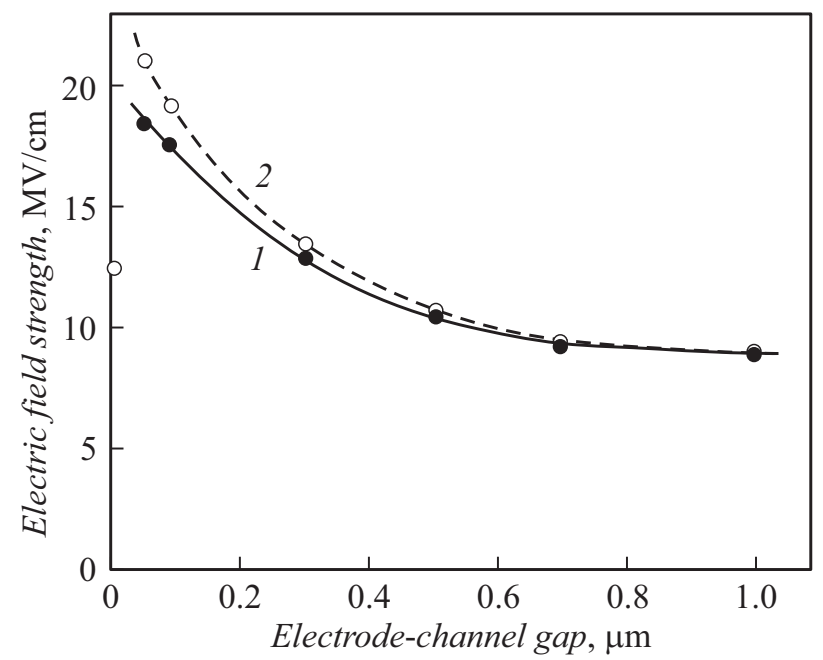

Pис. 6. Зависимость напряженности электрического поля у поверхности раствора от ширины диэлектрического зазора вокруг канала для $\Delta=0.02(1)$ и $\Delta=0.1 \mu \mathrm{m}$ (2).

1) поверхность раствора в канале плоская и 2) радиус ее кривизны равен радиусу канала.

Как видно, электрическое поле вблизи поверхности раствора неоднородно. Значения напряженности электрического поля непосредственно у поверхности раствора для приведенных случаев отличаются, хотя и не очень сильно.

Зависимость напряженности электрического поля у поверхности раствора от толщины электрода на вакуумной стороне мембраны показана на рис. 4 для двух значений ширины диэлектрического зазора между каналом и электродом.

В области толщин до $0.2 \mu \mathrm{m}$ напряженность поля несколько увеличивается с увеличением толщины электрода, тогда как при больших значениях зависимость практически отсутствует.
Несмотря на то что напряженность экстрагирующего ионы поля слабо зависит от толщины электрода, следует принять во внимание, что часть ионов, выходящих из раствора, испытывает столкновение с внутренними стенками электрода. Естественно ожидать, что доля ионов, которые избежали столкновений, падает при увеличении его толщины. Проведенная с помощью программы SIMION 8 симуляция движения ионов после их выхода из раствора показала, что при ширине диэлектрического зазора $\delta=0.22 \mu \mathrm{m}$ в области $\Delta>1.5 \mu \mathrm{m}$ менее половины выходящих из раствора ионов покидают границы интерфейса, не испытав соударений с внутренними стенками электрода (рис. 5).

Как показано на рис. 6, в области $\delta<0.5 \mu$ m напряженность электрического поля у поверхности раствора резко увеличивается при уменышении ширины диэлектрического зазора вокруг канала.

\section{Обсуждение результатов}

Следуя оценкам, проведенным в [1,2], будем считать, что эффективный выход ионов из раствора, заполняющего канал мембраны, происходит при напряженности электрического поля вблизи поверхности жидкости около $10 \mathrm{MV} / \mathrm{cm}$ и выше. Использование этого критерия позволяет в рамках используемой модели выбрать геометрические параметры интерфейса, которые должны обеспечить его эффективную работу.

Как видно из рис. 3, напряженность экстрагирующего ионы электрического поля зависит от кривизны поверхности жидкости. Радиус ее кривизны, согласно [3,5], определяется из того условия, что давление, обусловленное поверхностным натяжением, должно уравновешивать атмосферное давление и давление, обусловленное действием электрического поля. Оценки показывают, что для лавсановой мембраны с каналами диаметром около $60 \mathrm{~nm}$, заполненными водно-глицериновой смесью, радиус кривизны поверхности жидкости в полях порядка $10 \mathrm{MV} / \mathrm{cm}$ будет существенно больше, чем радиус канала. Поэтому полученные значения напряженности электрического поля у поверхности раствора для плоской и сферической поверхностей можно рассматривать как граничные, между которыми находится значение, соответствующее реальному случаю. Таким образом, при ширине диэлектрического зазора менее $0.5 \mu \mathrm{m}$ и напряжении между электродами $50 \mathrm{~V}$ напряженность электрического поля находится в интервале 8-11 MV/cm, т.е. практически совпадает с требуемой для эффективной эмиссии ионов, независимо от толщины электрода на вакуумной поверхности мембраны. Вместе с тем высокую эффективность работы интерфейса можно обеспечить, если доля ионов, испытывающих соударения с внутренними стенками электрода на вакуумной стороне мембраны, будет достаточно мала. Для выполнения этого условия толщина электрода не должна превышать $2 \mu \mathrm{m}$. 
Следует отметить, что в используемой модели не учитывается влияние поляризации материала мембраны на напряженность поля вблизи поверхности раствора, заполняющего канал мембраны. Поляризация диэлектрика приводит к некоторому уменьшению напряженности поля у поверхности раствора. Однако принимая во внимание, что для лавсана, используемого в качестве материала мембраны, диэлектрическая постоянная невелика $(\varepsilon \approx 3)$, это уменьшение не будет очень большим. Учет поляризации диэлектрика в зазоре между каналом и электродом на вакуумной поверхности мембраны, как отмечалось в [6], дает уменьшение напряженности экстрагирующего ионы поля не более чем в $1 / \varepsilon$ раз, т.е. не больше чем в 3 раза.

Эффект поляризации материала мембраны можно скомпенсировать за счет простого увеличения напряжения экстракции по сравнению с напряжением, использовавшимся в приведенных выше расчетах. При этом оно увеличится максимум до $150 \mathrm{~V}$, оставаясь существенно меньше напряжений, приводящих к появлению нежелательных вторичных процессов. Повидимому, предельные значения напряжений должны быть определены экспериментально с учетом электрической прочности зазора между электродом на вакуумной стороне мембраны и каналом, заполненным раствором.

Напряженность электрического поля вблизи поверхности жидкости может быть существенно увеличена также за счет уменьшения ширины диэлектрического зазора между каналом и электродом на вакуумной стороне мембраны - практически в 2 раза при уменьшении ширины зазора до $20 \mathrm{~nm}$. Однако создание таких наноразмерных зазоров вокруг каналов, распределенных по поверхности мембраны случайным образом, по-видимому, представляет собой достаточно сложную технологическую задачу.

Основным преимуществом рассматриваемого модифицированного интерфейса по сравнению с конструкцией, использующей металлическую сетку, расположенную вблизи вакуумной поверхности мембраны $[7,8]$, является то, что благодаря низкой энергии экстрагированных ионов можно ожидать практически полного отсутствия вторичных процессов, приводящих к генерации побочных ионов. Можно ожидать также, что эффекты, связанные с зарядкой вакуумной поверхности диэлектрической мембраны, не будут играть существенной роли в связи с тем, что бо́льшая ее часть будет покрыта проводящим электродом. Кроме того, важно, что в модифицированном интерфейсе все каналы находятся в эквивалентных условиях. В конструкции с сеткой напряженность экстрагирующего ионы электрического поля на разных участках мембраны может сильно различаться [6] она выше в тех местах, которые расположены ближе к образующим сетку металлическим прутикам, и ниже в тех местах, которые находятся ближе к центру окна сетки.

\section{Заключение}

Основным результатом настоящей работы является то, что на основании проведенных расчетов электрических полей в модифицированном мембранном интерфейсе удалось определить диапазон геометрических характеристик, которые могут обеспечить эффективную полевую экстракцию ионов из растворов при достаточно низких напряжениях. Вместе с тем реализация рассмотренной конструкции требует разработки нанотехнологий, позволяющих создавать на поверхности мембраны узкие диэлектрические зазоры вокруг каналов, распределенных случайным образом. Ожидается, что предлагаемая конструкция с электродом, расположенным непосредственно на вакуумной стороне мембраны, даст возможность получить ряд важных преимуществ по сравнению с использовавшимися ранее.

\section{Список литературы}

[1] Balakin A.A., Gridin V.V., Schechter I. // J. Phys. Chem. A. 1998. Vol. 102. N 47. P. 9470-9475. DOI: 10.1021/jp981942h

[2] Балакин А.А., Буйдо Е.А., Новикова Л.И. // ЖТФ. 2010. T. 80. Вып. 9. С. 118-124. [Balakin A.A., Buido E.A., Novikova L.I. // Tech. Phys. 2010. Vol. 55. N 9. P. 13511356.] DOI: $10.1134 / \mathrm{S} 1063784210090197$

[3] Yakovlev B.S., Talrose V.L., Fenselau C. // Anal. Chem. 1994. Vol. 66. N 10. P. 1704-1707. DOI: 10.1021/ac00082a017

[4] Balakin A.A., Dodonov A.F., Novikova L.I., Talrose V.L. // J. Electrostatics. 1997. Vol. 40-41. P. 615-620. DOI: org/10.1016/S0304-3886(97)00057-0

[5] Balakin A.A., Buido E.A., Golcova E.A., Novikova L.I., Talrose V.L. // J. Electrostatics. 2006. Vol. 64. N 7-9. P. 555 561. DOI: $10.1016 /$ j.elstat.2005.10.010

[6] Балакин А.А., Буйдо Е.А. // ЖТФ. 2013. Т. 83. Вып. 11. C. 130-135. [Balakin A.A., Buido E.A. // Tech. Phys. 2013. Vol. 58. N 11. P. 1675-1680.] DOI: $10.1134 / \mathrm{S} 1063784213110042$

[7] Балакин А.А., Хидиров С.Г. // ЖТФ. 2014. Т. 84. Вып. 11. C. 134-140. [Balakin A.A., Khidirov S.G. // Tech. Phys. 2014. Vol. 59. N 11. P. 1711-1717.] DOI: $10.1134 / \mathrm{S} 1063784214110048$

[8] Балакин А.А., Хидиров С.Г., Буйдо Е.А. // ЖТФ. 2016. T. 86. Вып. 10. С. 32-37. [Balakin A.A., Khidirov S.G., Buido E.A. // Tech. Phys. 2016. Vol. 61. N 10. P. 1474-1479.] DOI: $10.1134 / \mathrm{S} 1063784216100054$

[9] Балакин А.А., Буйдо Е.А., Хидиров С.Г. Патент на изобретение № 2537961. Приоритет: 15.05.2013. Бюл. № 1. 10.01.2015.

[10] Manura D., Dahl D. SIMION (R) 8.0 User Manual. Scientific Instrument Services, Inc. Ringoes, NJ 08551, January 2008. http://simion.com/ 